Doug Geisler, Eva K. Grebel, and Dante Minniti, eds.

\title{
HST and Keck Observations of a Young Globular Cluster in the Spiral Galaxy NGC 6946
}

\author{
Søren S. Larsen and Jean P. Brodie \\ Lick Observatory, University of California at Santa Cruz, USA
}

Bruce G. Elmegreen

IBM Research Division, T.J. Watson Research Center, New York, USA

Yuri N. Efremov

Sternberg Astronomical Institute, Moscow, Russia

Paul W. Hodge

Astronomy Department, University of Washington, Seattle, USA

Tom Richtler

Grupo de Astronomía, Universidad de Concepción, Concepción, Chile

\begin{abstract}
Using new (Cycle 9) HST / WFPC2 data, we have studied a peculiar star forming region in the nearby Sc-type spiral NGC 6946. The region has a bubble-like shape and a diameter of about 600 pc. Near the centre is an extremely luminous young globular cluster with $M_{V}=-13.2$ mag. The cluster has a compact core with a core radius of about 1.3 pc but is surrounded by an extended halo with a power-law luminosity profile similar to that observed for young LMC clusters. From Keck / HIRES high-dispersion spectroscopy we measure a velocity dispersion of $10 \mathrm{~km} \mathrm{~s}^{-1}$, leading to a dynamical mass estimate of about $1.7 \times 10^{6}$ solar masses. The dynamical mass estimate is comparable to the expectation from population synthesis modelling under the assumption of a Salpeter IMF extending down to $0.1 \mathrm{M}_{\odot}$.
\end{abstract}

\section{Introduction}

During a search for young massive clusters in a sample of nearby spiral galaxies, Larsen \& Richtler (1999) encountered a peculiar star forming region in NGC 6946. First noted by Hodge (1967), it has a bubble-like appearance and contains dozens of young star clusters and field stars within a radius of about 300 pc, all with an age of about $15 \mathrm{Myr}$ (from $U B V$ colours, Elmegreen, Efremov and Larsen 2000). It is located in a spiral arm at about $5 \mathrm{kpc}$ from the centre of NGC 6946. Within the bubble is an extremely luminous star cluster with $M_{V}=-13.2 \mathrm{mag}$. In this poster paper we discuss imaging of the cluster with 
the HST Planetary Camera and high-dispersion spectroscopy from the HIRES spectrograph on the Keck I telescope.

\section{Structure and dynamical mass of the cluster}

The bubble-like shape of the star forming region is readily visible in Figure 1 , showing the PC field of view. The young star cluster is the single most luminous object in the image, near the centre of the region. The spatial profile of the cluster is well resolved on the HST image and we estimate that it has a core radius of about $1.2 \mathrm{pc}$. The outer parts of the profile are well fitted by a power-law. The cluster light gradually merges with the background and the half-light radius is not well-defined, but from the HST image we estimate it to be about 13 pc (Larsen et al. 2001).

In order to obtain a dynamical mass estimate for the cluster, Keck / HIRES spectra were obtained in August 2000. The spectra have a resolution of $\lambda / \Delta \lambda \sim$ 40000 , sufficient to derive a velocity dispersion for the cluster using a crosscorrelation technique similar to that employed by Ho \& Filippenko (1996). We thus find a velocity dispersion of $\sigma_{x}=10.0 \pm 2.7 \mathrm{~km} \mathrm{~s}^{-1}$ for the cluster. By use of the virial theorem, this would imply a total virial mass of $M=7.5 \sigma_{x}^{2} r_{h} / G=$ $3 \times 10^{6} \mathrm{M}_{\odot}$, where $r_{h}$ is the 3-dimensional half-mass radius (about 1.3 times the projected half-light radius). However, because of the ill-determined half-light radius we decided to carry out a more detailed modelling of the light profile of the cluster, assuming that the cluster is in hydrostatic equilibrium (see Larsen et al. 2001 for details). From this modelling and the measured velocity dispersion we obtain a lower dynamical mass estimate of $(1.7 \pm 0.9) \times 10^{6} \mathrm{M}_{\odot}$ inside a radius of $65 \mathrm{pc}$, corresponding to the aperture radius used for the cluster photometry.

For a cluster age of $15 \mathrm{Myr}$ and $M_{V}=-13.2 \mathrm{mag}$, Bruzual \& Charlot (1996) population synthesis models predict a mass of $8.2 \times 10^{5} \mathrm{M}_{\odot}$ for a Salpeter IMF extending down to $0.1 \mathrm{M}_{\odot}$. Thus, our dynamical mass estimate is somewhat higher than the prediction, but consistent with the Salpeter IMF within the uncertainties. However, a significant lack of low-mass stars is ruled out. It therefore appears likely that the cluster will remain bound, eventually evolving into an object resembling the old globular clusters observed in the halo of our own and other galaxies.

\section{Conclusions}

With a total mass somewhere around $10^{6} \mathrm{M}_{\odot}$, this is probably the most massive young star cluster yet encountered in the disk of a normal spiral galaxy. It is comparable in mass and luminosity to the brightest young clusters in the Antennae merger galaxies, and provides a striking piece of evidence that "super" star clusters can form not only in mergers and starbursts, but also in the spiral arms of normal galaxies. The surroundings of the clusters are, however, unusual (see also Efremov, this volume).

Acknowledgments. This work was partially supported by NSF grant AST9900732 and by a travel grant from the American Astronomical Society. 


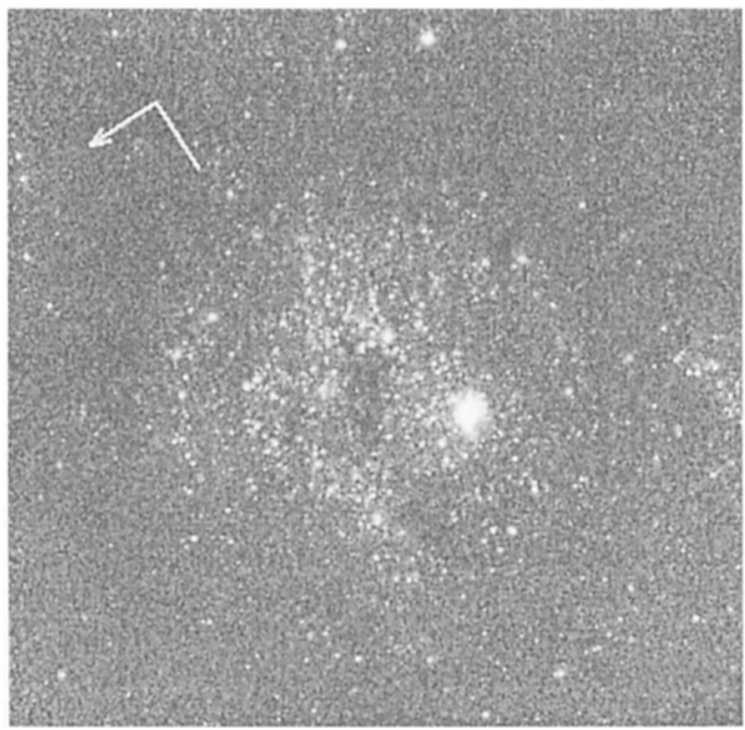

Figure 1. The HST Planetary Camera field of view, showing the young globular cluster (near the centre) and the surrounding star forming region. At the distance of NGC 6946, the field is about $1 \mathrm{kpc}$ across. The arrow is pointing towards the North.

Support for program number GO-8715 was provided by NASA through grants GO-08715.02-A and GO-08715.05-A from the Space Telescope Science Institute.

\section{References}

Bruzual, G. A. \& Charlot, S. 1996, unpublished

Elmegreen, B. G., Efremov, Y. N., Larsen, S. S. 2000, ApJ, 535, 748

Ho, L. C. \& Filippenko, A. V. 1996, ApJ, 472, 600

Hodge, P. W. 1967, PASP, 79, 29

Larsen, S. S., \& Richtler, T. 1999, A\&A, 345, 59

Larsen, S. S., Brodie, J. P., Elmegreen, B. G. et al. 2001, ApJ, 556, 801 